\title{
SOAP: A Cognitive Hybrid Routing Protocol for Mobile Ad-hoc Networks
}

\author{
Amina Al-Rokabi \\ WMN (Wireless Multimedia \& Networking) Research \\ Group \\ Faculty of Science, Engineering and Computing \\ SEC, Kingston University \\ London, United Kingdom \\ k1262562@kingston.ac.uk
}

\author{
Christos Politis \\ WMN (Wireless Multimedia \& Networking) Research \\ Group \\ Faculty of Science, Engineering and Computing \\ SEC, Kingston University \\ London, United Kingdom \\ C.Politis@kingston.ac.uk
}

\begin{abstract}
With the shift towards wireless technology increasing at a faster rate than ever before, it is becoming ever more important to focus on optimising the routing functionality of a wireless network. The purpose of this paper is to outline the different types of routing that can be applied in a Mobile Ad Hoc Network (MANET) and to propose a new routing protocol named SOAP. The SOAP routing protocol is designed as a cognitive hybrid protocol, making use of the functionality that exists in the popular proactive OLSR (Optimized Link State Routing) and reactive AODV (Ad hoc On-Demand Distance Vector) MANET routing protocols. The paper concludes that the SOAP protocol brings about performance improvements across a few Quality of Service metrics such as a lower delay than AODV and a lower routing overhead than OLSR but more work needs to be placed in ensuring its reliability and consistency across different network conditions. The SOAP protocol did not perform consistently in terms of packet loss and throughput and these issues should be addressed in further research.
\end{abstract}

Keywords-Routing protocols, Mobile Ad Hoc Network (MANET),cognitive, hybrid, proactive, reactive, OLSR, AODV, SOAP

\section{INTRODUCTION}

Wireless communication is now at the forefront of research and commercial advancement and is playing an extremely important role in influencing how we harness the technology that has been available to us for many years. The next generations of wireless communication systems bring about a need for rapid deployment of independent agents in a network.

Many scenarios such as emergency rescue operations and disaster relief where a centralized network is not possible due to the lack of infrastructure can be treated as applications of a Mobile Ad Hoc Network. In these networks, each node participates in the provision of reliable operations in the network. The range of applications for MANET is very diverse and each type of usage brings its own challenges and constraints. For example, a small network of static nodes such as a group of laptops operating on a mesh network in a developing country will be constrained by power sources and therefore the design of the network protocol will need to minimize power consumption as much as possible.

Routing is one of the most important aspects of MANET as all network activity including discovering the topology and delivering messages needs to be carried out by the nodes themselves. There are two main approaches to this: proactive routing and reactive routing. Proactive or table-driven routing maintains an up to date list of destinations and routes by distributing routing tables periodically throughout the network, resulting in a major overhead due to the amount of data being transmitted throughout the network and the time taken for changes in the network to be realised. Reactive or on-demand routing on the other hand finds routes on demand by flooding the network with request packets known as RouteRequests, resulting in longer latency due to the time taken for routes to be found and the potential for routing overhead when frequent route discoveries are required.

Due to the nature of the applications of MANET, such as emergency rescue operations and disaster relief, it is of crucial importance to find a way to minimize and reduce the problems associated with MANET routing. This research focuses on a third approach to routing known as the hybrid approach which combines the advantages of both proactive and reactive routing. This will result in the design of a protocol that is geared towards efficiency and performance in the applications of technology that need it the most.

The paper introduces the concept of MANET and its different use cases. It then goes on to give an overview of the different types of routing protocol, starting with a detailed look at the proactive OLSR routing protocol followed by the reactive AODV routing protocol and finally a selection of popular hybrid routing protocols including SHARP, ZRP and CML. The SOAP routing protocol is then introduced and its algorithm is defined. The simulation itself is then outlined along with the results and their analysis. The paper then concludes that the SOAP protocol brings about performance improvements across a few Quality of Service metrics such as a lower delay than AODV and a lower routing overhead than OLSR but more work needs to be placed in ensuring its 
reliability and consistency across different network conditions. The SOAP protocol did not perform consistently in terms of packet loss and throughput and these issues should be addressed in further research.

\section{MOBILE AD-HOC NETWORK (MANET)}

\section{A. Introduction to MANET}

There are two main types of networks: infrastructure networks such as base-stations in cellular networks and infrastructure-less networks such as Mobile Ad hoc Networks (MANET). A Mobile Ad hoc Network (MANET) consists of multiple mobile nodes which share an open transmission medium and the same available bandwidth.

Nodes in MANET are autonomous, self-organized and selfconfigured with no need for any pre-existing infrastructure or any centralized control system and have the ability to be immediately deployed when required. Each node in MANET acts as both an end system and a router by forwarding data packets through the network

\section{MANET applications}

In extreme circumstances like forest fires, earthquakes, floods and terrorist attacks, the ability to construct a network topology immediately has a significant effect on how well the rescue teams are able to co-ordinate with each other. An emergency MANET (eMANET) is ideal for such scenarios because it is based on protocols that behave in a distributed way, allowing a quick response to rapid changes in network topology such as nodes joining or leaving the network. MANET technology is also utilised commercially through electronic payment systems and vehicle-to-vehicle communication, for example. It also has many tactical applications including military communications in situations that require utmost secrecy and reliability. [1]

\section{Challenges of MANET}

The unpredictability of the environment, unreliability of the wireless device, resource constraints on the nodes, transmission errors, node failures, link failures, route breakages, and congested nodes or links are major challenges in MANET [2]. Most of the nodes that are part of a MANET often rely on batteries or other exhaustible means for their energy. For nodes like this, the most important criteria when designing a fully optimised system may be energy conservation [3]. As nodes in a MANET are distributed to an area that is greater than the radio scope of an individual node, routing protocols are required to facilitate the connectivity between nodes that are on different radio ranges [4], meaning less physical security and therefore a higher chance of eavesdropping, spoofing and denial-of-service attacks.

\section{B. MANET Routing Protocols}

In order to trace topological changes in MANET networks, several approaches can be taken depending on the type of routing protocol. These routing protocols fall into three general categories:
- Flat routing

- Hierarchical routing

- Geographic position assisted routing

A flat routing protocol uses an address scheme meaning each node that contributes to the routing has a similar role. A hierarchical approach, however, assigns different roles to the nodes that contribute to the routing.

Geographic position assisted routing approaches provide each node with a Global Positioning System (GPS) device. Achieving this is plausible in the current climate as GPS devices are cheap and are able to provide very good precision, making it possible to provide location information with a precision of a few meters. In the same way that information on the location of nodes is used for directional routing in MANET networks, the universal clock allows for global synchronization among nodes equipped with GPS devices.

The performance of a routing protocol can be improved through the use of location information but it should be noted that this location information can be unreliable by the time it is used due to constant location changes. As each node needs to be equipped with A GPS device, this results in the additional cost of each node having to calculate and update its geographic location periodically. The Location Aware Routing (LAR) and LANMAR protocols are examples of protocols that use GPS information to determine the location of nodes [5].

Flat routing protocols are divided into three main types: proactive, reactive and hybrid. In this thesis, we will focus in more detail on these three types of flat routing protocol.

\section{Proactive Routing Protocols}

Each node in a MANET network which uses a proactive, table-driven routing protocol maintains coherent and consistent routing information for the purpose of finding routes to every other possible node within the network. Nodes exchange control messages regularly between themselves in order to update their routing tables and respond immediately to any topology change.

The most well-known MANET proactive protocol is Optimized Link State Routing Protocol (OLSR) RFC3626 and is one of the most researched protocols [6]. In the OLSR protocol, route discovery and route maintenance is enabled by the periodic exchange of route information. Therefore, each node stores information about all nodes regardless of whether this information is utilised. Thus, OLSR achieves its highest efficiency in small dense networks.

Classic link state routing algorithms normally suffer from control packet flooding, OLSR minimises this as each node in the network independently chooses its own set of symmetric one-hop neighbour nodes, known as Multi Point Relays or the MPRset, to forward its generated link state packets through. As a node discovers all its routes to destinations through its MPRset members only, the efficient performance of OLSR comes down to the selection of the MPRset nodes. 
OLSR has three kinds of control message: Hello (H) messages, Topology Control (TC) messages and Multi Interface Declaring (MID) messages. Each node exchanges hello messages with its one-hop neighbours periodically for link sensing, detecting neighbours, MPR signaling tasks and selection of multipoint relays. MPR nodes forward TC messages generated by their MPR selector nodes to the whole network periodically in order to declare their advertised link set. Nodes that have multiple OLSR interfaces use MID messages to declare their interfaces information to all network nodes using the MPR technique.

\section{Reactive Routing Protocols}

On-demand, distance vector reactive routing protocols only establish end-to-end connection routes when a node requires communication to a particular destination and it doesn't know the route to it. This approach may cause longer delays than proactive routing as path discovery is initiated each time a connection is needed [7]. The on-demand reactive approach is initiated by reactive mechanisms and does not broadcast control messages periodically like the proactive approach. The well-known reactive Ad-hoc On Demand Distance Vector Routing Protocol (AODV) protocol RFC 3561[8] is a good example of a reactive routing approach.

Discovering routes and maintaining routes are the two main phases of AODV. In AODV routes are only established when needed, as every node in the network maintains a traditional routing table which stores route information about many destination nodes with one entry per destination. AODV nodes exchange Hello messages periodically with neighbours to inform each other about their presence and to activate the links between them. AODV nodes discover routes by using queryresponse cycles and there are three kinds of control messages: Route Request (RREQ), Route Reply (RREP) and Route Error (RERR) and all these messages are sent via UDP.

When the route between a source node and destination node is unknown, the source node transmits a route request (RREQ) message to all of its neighbours. When each intermediate node that is between the source node and destination node receives a RREQ message, it keeps a reverse path to the node it received the RREQ message from in its routing table before processing the RREQ message. This is in order to redirect the route reply (RREP) packet in a hop-by-hop style back to the node which initiated the RREQ. A node monitors the link statuses of the next hop in active routes and when it detects a link break in an active route it notifies other nodes about this link break by sending a RERR message stating which destinations are invalid due to the loss of the link.

\section{Hybrid Routing Protocols}

As network size, density and the level of node mobility may vary unpredictably, there isn't a particular routing protocol suited to all network features and application behaviours. Hybrid routing protocols utilise the positive features of tabledriven proactive routing protocols and the positive features of on-demand reactive routing protocols depending on certain conditions.
The authors in [9] proposed a hybrid routing protocol known as the Zone Routing protocol (ZRP), which creates a zone that surrounds each node encompassing all of its neighbours. Proactive routing is applied inside the zone and is termed Intra-zone Routing Protocol (IARP). Reactive routing is carried out between nodes in different zones and is termed Inter-zone Routing Protocol (IERP) [9]. The aim of the ZRP hybrid approach is to reduce the proactive routing control overhead as well as the reactive routing latency associated with route discovery.

Other hybrid approaches are designed based on group mobility in MANET such as the LANMAR hybrid protocol [10]. For each group of nodes that are likely to move within the network together, a node is selected as a landmark node. There is a defined range called a scope so that each node in the network should ideally be within the scope of its designated landmark node.

Sharp Hybrid Adaptive Routing Protocol (SHARP) [11] is another hybrid MANET routing protocol. It differs from ZRP in that it creates zones around destinations that are considered popular based on the number of sources it receives data from. This is achieved by dynamically adapting the zone radius for each destination node based on the incoming data traffic and the level of mobility in the network. In this case, SHARP reduces control overhead but may suffer from a higher end-toend delay and packet loss rate as the zone's radius varies depending on the network conditions.

The majority of hybrid routing protocols define the proactive and reactive components regardless of the network condition. The authors of [12] tackled this and proposed a hybrid routing protocol named ChaMeLeon (CML) which adapts to the network size and switches between a proactive approach that uses the OLSR protocol and a reactive approach that uses the AODV protocol.

\section{OUR PROPOSED COGNITIVE HYBRID ROUTING PROTOCOL (SOAP)}

In this section we propose SOAP (Simplified OLSR \& AODV Protocol), a cognitive hybrid routing protocol for Mobile Ad-Hoc networks that has both proactive and reactive characteristics. The proactive element of the protocol will be a modified version of the OLSR routing protocol and the reactive element will be the AODV routing protocol. The SOAP protocol can be better explained by dividing its core functionality into two main parts. The first part is the definition of a modified version of the OLSR routing protocol to be named "S-OLSR" that closely resembles the OLSR routing protocol with two main differences.

The second part is the definition of a logical component to be named "SOAP Helper" that acts as a layer in front of the SOLSR and AODV routing protocols that is responsible for delegating the task of routing to the appropriate routing protocol as shown in figure 1: 


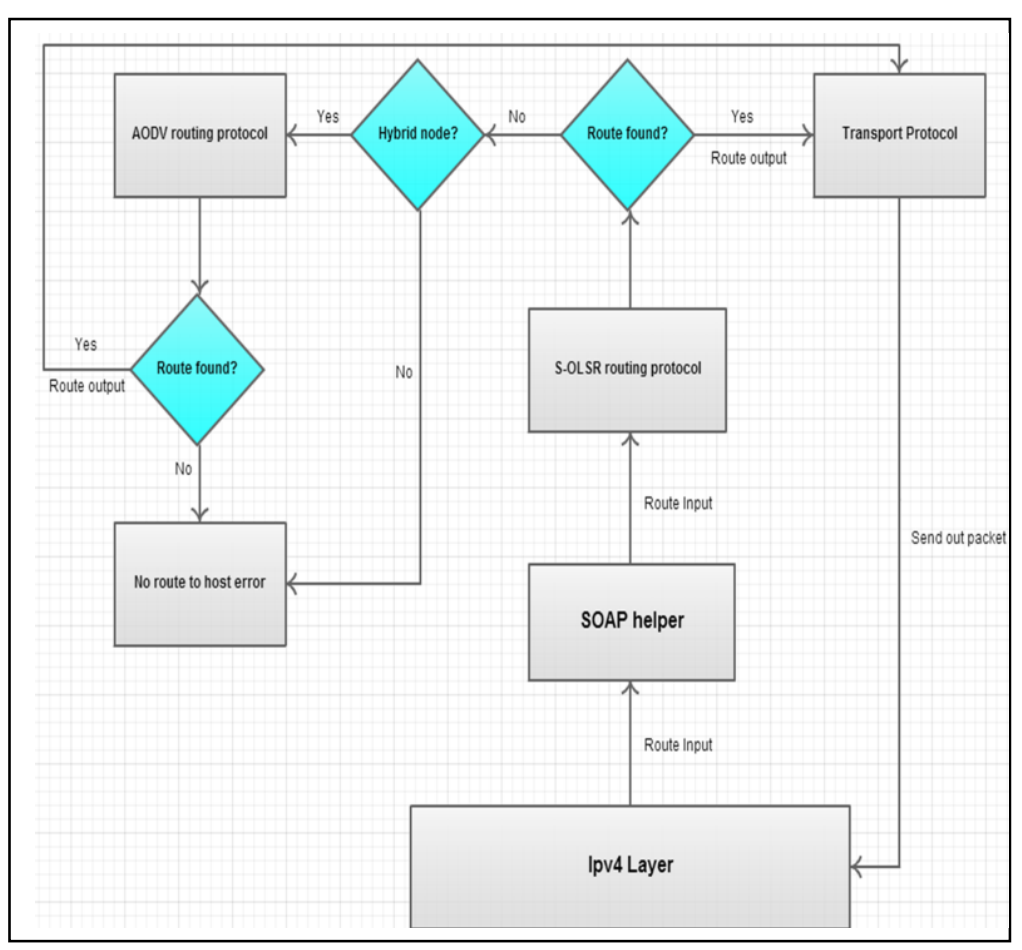

Fig. 1. System flow diagram

\section{A. S-OLSR}

This is a version of the OLSR routing protocol that has had two modifications made to it: The first modification is to decrease the number of HELLO messages that are sent periodically. This can be achieved by increasing the HELLO interval defined in the routing protocol. The original OLSR routing protocol sets its HELLO interval with a default of 2 seconds. This means that after every 2 seconds each node broadcasts a HELLO message to all one hop neighbours. The S-OLSR routing protocols increases this value and sets a HELLO interval with a default value of 3 seconds. This in effect reduces the number of HELLO messages that are broadcasted to all one hop neighbours by a third in comparison with the original OLSR routing protocol.

The second modification is to disable the sending of Topology Control(TC) messages completely. The original OLSR routing protocol uses TC messages to describe link states to neighbour nodes. TC messages are sent periodically and are also generated immediately when a change is detected in the MPR selector set. This functionality is removed in SOLSR in a bid to reduce each node's global understanding of the network topology and allow it to focus on its local topology. Therefore, a node will build up its knowledge of the local topology proactively using S-OLSR. When a node is required to route a packet outside of its local topology, the SOAP helper component comes into play as discussed below.

\section{B. SOAP Helper}

This component sits in front of the proactive S-OLSR routing protocol and the reactive AODV routing protocol in order to achieve the desired cognitive hybrid behaviour. It is responsible for delegating the task of finding a route by consulting the S-OLSR and AODV routing protocols one after the other. Each routing protocol maintains its own routing table and if the route for a packet cannot be found using the S-OLSR routing protocol, the AODV routing protocol is used instead if the node is a cognitive hybrid node.

A cognitive hybrid node is defined as a node with at least one MPR selector and therefore an MPR node. In the same way that only MPR nodes are used to retransmit packets in classical OLSR, the SOAP protocol restricts the ability of a node to behave in a cognitive hybrid way to this subset of nodes. The MPR nodes within the network are chosen using the Dijkstra's shortest path algorithm in the same way as the OLSR routing protocol. This is to reduce the overhead that would come about if all nodes were able to act in a cognitive hybrid fashion. SOAP differs from other hybrid protocols, from what can be seen reading related literature, in that it does not just switch between the pure proactive and reactive approach.

The SOAP protocol aims to reduce the overhead associated with OLSR as a result of TC messages and at the same time by making more use of MPR nodes by allowing them to behave in a cognitive hybrid fashion. In theory, comparing the SOAP hybrid protocol with the original pure proactive OLSR protocol, there is no need for a node to have global knowledge of the whole topology. It does not need to advertise its routes to the rest of the network by sending topology control packets periodically, and thus reduces the overall routing overhead associated with pure proactive routing.

Furthermore, fewer resources such as bandwidth and power are consumed as each node has to only maintain a routing table of local topology and not the entire network. SOAP differs from other hybrid protocols, from what can be seen reading related literature, in that it does not just switch between the pure proactive and reactive approach.

The SOAP protocol aims to reduce the overhead associated with OLSR as a result of TC messages and at the same time by making more use of MPR nodes by allowing them to behave in a hybrid fashion.

In order to test the SOAP protocol, a network simulation will be carried out for nodes implementing the SOAP protocol and the performance will be compared with nodes implementing the OLSR protocol on its own and the AODV protocol in its own. The comparison will be carried out by comparing the following routing metrics which are normally analyzed when evaluating a MANET protocol [13]: Throughput, End-to-end delay, Packet loss ratio, and Routing Overhead,

\section{SIMULATION ENVIRONMENT}

A network simulator is a piece of software that aims to mimic the behaviour of a real computer network as accurately as possible. As network simulation software does not actually involve utilising the functionality of a real network, the components of a network such as the physical layer, channels, devices, sockets, and packets are modelled using abstract software principles. There are many network simulators available, each with their advantages and disadvantages.

The chosen network simulator was NS3 [14], which ships with OLSR built in and is very straight forward to install on a Linux distribution. The documentation is very thorough and the 
online discussion groups are active and are often frequented by NS3 core developers. Each module ships with tests and example simulations which allows for a more structured and positive learning experience. Performance analysis can be carried out using NS3's built-in FlowMonitor or DataCollector packages or by external tools such as Wireshark.

\section{A. Simulation}

The simulation was written in $\mathrm{C}++$, Table 1 gives an overview of the different simulation parameters:

TABLE I. SimULATION PARAMETERS

\begin{tabular}{|l|l|}
\hline \multicolumn{1}{|c|}{ Parameter } & \multicolumn{1}{|c|}{ Value } \\
\hline Simulation time & 50 seconds \\
\hline CBR data rate & $1500 \mathrm{kbps}$ \\
\hline CBR packet size & 64 bytes \\
\hline Physical mode & Dsss Rate 11Mbps \\
\hline Node speed & $1.5 \mathrm{~m} / \mathrm{s}$ \\
\hline Node pause time & 10 seconds \\
\hline Node Transmission power & $7.5 \mathrm{dBm}$ \\
\hline Wifi standard & $802.11 \mathrm{~b}$ \\
\hline Network size & $1000 \mathrm{x} 1000 \mathrm{~m}$ \\
\hline Mobility Model & Random Waypoint Mobility \\
\hline Number of nodes & $5,10,15,20,25,30,35,40,45,50$ \\
\hline Number of CBR connections & CHAIN* 12, 14, 17, 19, 22, \\
\hline & \\
\hline & \\
\hline & \\
\hline
\end{tabular}

This is an NS3 list routing protocol that tries OLSR first and then AODV

\section{B. Results gathering and calculation methods}

The ns3::FlowMonitor package was used to gather statistics for each network flow that was created during each simulation. The statistics that are provided by the ns $3:$ :FlowMonitor class include the total received bytes, received packets, transmitted bytes and transmitted packets for each flow.

It also provides a time value for the jitter sum and for the delay sum and specifies the number of lost packets in each flow.

\section{Results}

\section{Throughput}

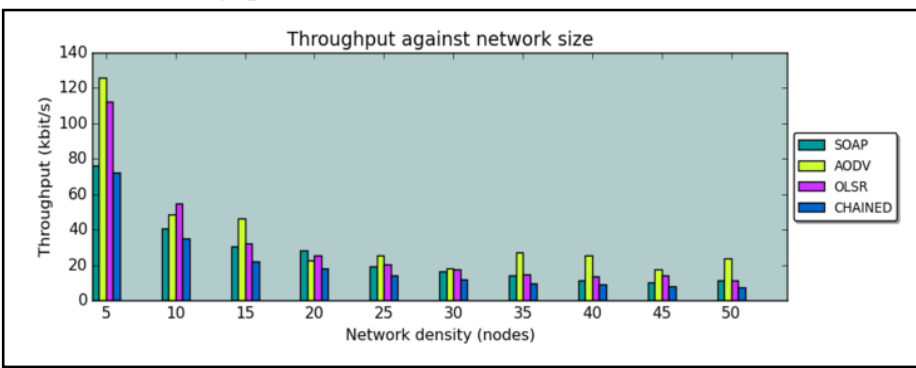

Fig. 2. Throughput for each routing protocol against network density

The results for throughput in figure 2 show that AODV generally had the highest throughput for more dense networks (35 - 45 nodes) and that OLSR had the highest throughput for less dense networks (10 nodes). The throughput values for SOAP were very similar to OLSR for denser networks (35-50 nodes) but were less than OLSR and AODV for less dense networks (5-10 nodes). The reason for the decrease in throughput across all routing protocols as the network density increases can be attributed to higher contention and in turn, a lower throughput for each node [15]. The lack of TC messages in the SOAP routing protocol may be responsible for it having a lower throughput in less dense networks but this optimization yields a throughput value that is no worse than OLSR in denser networks, therefore resulting in a fair trade-off.

\section{End to End delay}

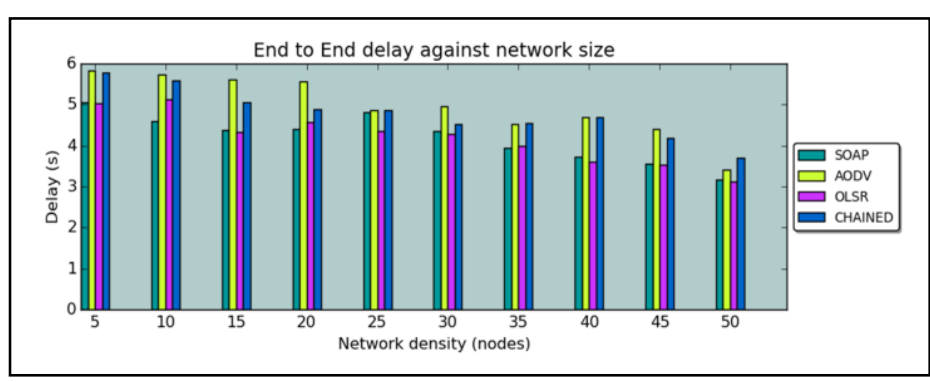

Fig. 3. End to end delay for each routing protocol against network density

The results for end-to-end delay in figure 3 show that AODV generally has a significantly higher delay over the SOAP and OLSR routing protocols. The reason for this high delay is that AODV's use of on-demand and reactive route discovery means that it needs to discover the route each time it needs to send data [16]. The results show that SOAP and OLSR only have a similar delay across the larger network densities ( 30 and 50 nodes). This can be explained by the fact that OLSR TC messages contribute to a single node's knowledge of the global topology and as these are not present in the SOAP protocol, delays will be incurred in networks that have low densities.

The results, however, also show that the SOAP protocol performs better than all other routing protocols when the number of nodes in the network is low (5-20). This appears to be an optimal network density, possibly due to the number of MPR nodes that are selected in this network density. The number of MPR nodes selected dictates how many nodes have 
the ability to use AODV but also affects the performance of the OLSR protocol. The results also show that the chained protocol has a higher delay than SOAP throughout as it will always fall back to AODV if the route is not found using OLSR.

\section{Packet loss ratio}

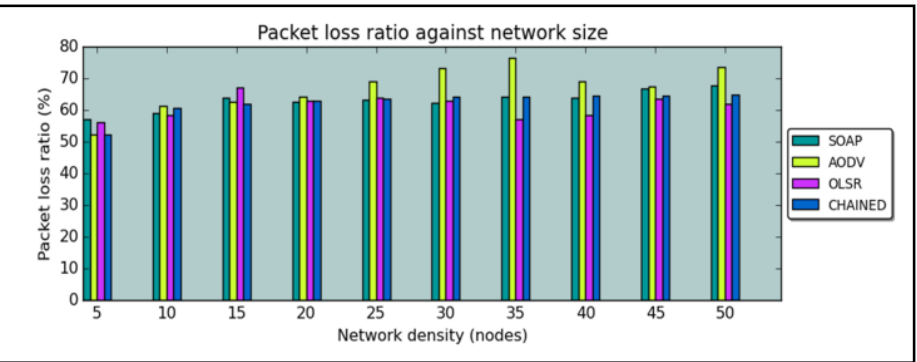

Fig. 4. Packet loss ratio for each routing protocol against network density

The results for packet loss in figure 4 show that OLSR has a lower packet loss ratio than all other routing protocols in more dense networks ( 35 - 50 nodes). The results also show OLSR has a lower packet loss ratio than AODV, in particular for every network density except the lowest (5 and 15 nodes). It can be seen from the results that the packet loss ratio is quite consistent for OLSR across different network densities, with a difference of about $5 \%$ between a network with 5 nodes and a network with 50 nodes. This is not the case for the SOAP and AODV routing protocols, with a variation of $11 \%$ and $21 \%$ respectively.

It is worth noting that the packet loss ratio was considerably high across all simulations and this was because of the high CBR data rate used in the simulation (1500kbps), meaning that the majority of the lost packets were down to high congestion. The packet loss ratio is an indicator of the reliability of a protocol and OLSR appears to be consistent in its reliability across different network densities. This can be explained by the fact that OLSR uses MPR nodes that are always available to forward data regardless of the network density or structure, ensuring packets are delivered consistently. This is not the case for AODV as it can be seen that the packet loss ratio increases in a linear fashion as the network density increases.

The SOAP protocol has a similar packet loss ratio to OLSR for small networks ( 5 - 10 nodes) but the difference between the two protocols increases in a linear fashion as the network density increases. Comparing SOAP and AODV, the opposite effect can be seen, with the AODV packet loss being higher than SOAP in more dense networks ( 25 nodes onwards).

Both of these patterns can be explained by the fact that as the network density increases, there are fewer cognitive hybrid nodes present compared to normal nodes as the number of MPR nodes is less than the number of normal nodes. As a cognitive hybrid node is more reliable and more likely to find a route, the number of cognitive hybrid nodes will affect the overall reliability of the protocol.

This theory is further supported by the performance of the CHAINED protocol, which had the lowest packet loss ratio across all simulations.

\section{Routing overhead}

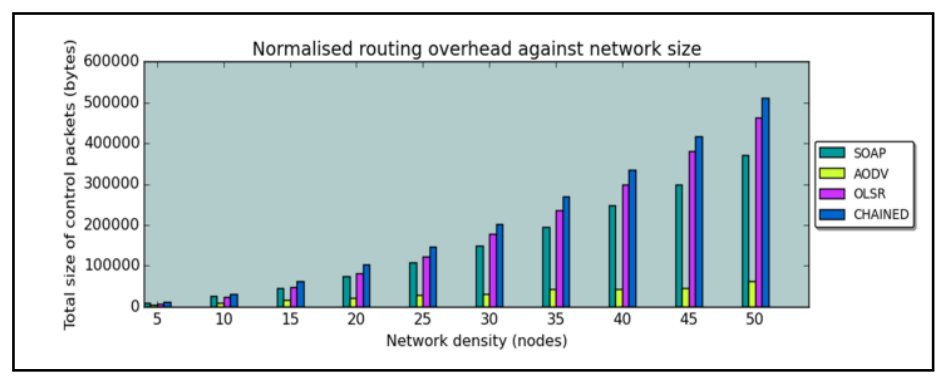

Fig. 5. Routing overhead for each routing protocol against network density

The results for routing overhead in figure 5 show a very consistent pattern across all network densities for all routing protocols. This consistency is due to the fact that the routing overhead is determined by the design of the routing protocol and is not affected by network factors such as link breakage and congestion, like the other metrics. The results show that AODV has the lowest routing overhead across all network densities. This is because of the reactive nature of the protocol, meaning that routing packets are only sent where necessary unlike OLSR which requires every node to send periodic messages. The routing overhead for the SOAP protocol is lower than OLSR as SOAP makes use of S-OLSR which does not use TC messages and sends HELLO messages less frequently. The CHAINED protocol performs the worst out of all the others with the highest routing overhead across all network densities. This is because it bears the overhead of both the AODV and OLSR protocols put together as it consults both routing protocols.

\section{CONCLUSION}

The SOAP protocol was designed to tackle the issues associated with pure proactive and reactive protocols by utilising both the proactive elements of the OLSR routing protocol as well as the reactive elements of the AODV routing protocol. A more optimised version of the OLSR routing protocol was implemented (S-OLSR) by reducing the number of HELLO messages sent and stopping the transmission of TC messages. The increased the likelihood of packets being lost when using this optimised version of OLSR and was addressed by allowing some of the nodes to fall back to the AODV routing protocol when no route could be found. The selection process for these nodes made use of the same algorithm employed by the OLSR routing protocol when selecting multipoint relay (MPR) nodes. This was to ensure that only the best candidates are selected to behave in a cognitive hybrid fashion and to reduce the overheads and delays associated with using more than one routing protocol. A simulation was carried out to model a typical scenario in a mobile ad hoc network with many nodes moving constantly within a fixed area.

The results presented in section IV indicate that the SOAP routing protocol brings about a mixed level of improvement in performance across the different quality of service metrics. The results for the routing overhead showed that the SOAP protocol had a lower routing overhead than the OLSR routing protocol. The results also showed that the SOAP protocol had lower delays than all other routing protocols in less dense networks. Packet loss was generally the same between the SOAP protocol 
and OLSR except for more dense networks where SOAP had a higher packet loss ratio. The results for throughput, however, showed that SOAP had a lower throughput than AODV and OLSR.

The results prove that the SOAP protocol resulted in improvements across some of the performance metrics such as a lower delay than AODV and a lower routing overhead than OLSR. However, more research is required into making these improvements consistent and reliable across different network densities. The SOAP protocol did not perform consistently in terms of packet loss and throughput and these issues should be addressed in further research. The SOAP protocol can be changed to take the network density into account when turning a node into a cognitive hybrid node. This allows for a more robust implementation that adapts well to network conditions. In addition to this, the S-OLSR routing protocol can be improved by re- introducing TC messages but restricting the transmission of these messages. This will still result in a reduction of the total routing overhead but will ensure the benefits of MPR nodes are fully realised.

In conclusion, more work needs to be invested in assessing the impact of factors such as node speed, node power and node positioning on the performance of the different routing protocols. It is also important to compare the performance of the SOAP routing protocol with other hybrid routing protocols to assess its potential use in a production environment. This paper was successful in designing and implementing a cognitive hybrid approach for routing traffic in a mobile ad hoc network (MANET). However, more work needs to be done to ensure that the performance improvements are more consistent across all metrics and different network conditions.

\section{ACKNOWLEDGMENT}

The authors would like to thank Fariborz Entezami for his assistance during the result gathering and analysis process.

\section{REFERENCES}

[1] I.F. Akyildiz, X. Wang, W. Wang. 2005. "Wireless mesh networks: a survey." Comput. Netw, vol. 47, no. 4, pp. 445-487, March 2005

[2] P.S. Kumar and S. Ramachandram, "Comparative Performance Analysis of GZRP and AOMDV in MANETs," Information Technology for Real World Problems (VCON), 2010 Second Vaagdevi International Conference on , vol., no., pp.52,56, 9-11 Dec. 2010

[3] S. Corson and J. Macker, "Mobile Ad hoc Networking (MANET): Routing Protocol Performance Issues and Evaluation Considerations," (2501) , Internet Engineering Task Force , IETF , RFC 2501 (Informational) (1999).

[4] P. Jacquet, P. Muhlethaler, T. Clausen, A. Laouiti, A. Qayyum, L. Viennot, "Optimized link state routing protocol for ad hoc networks," Multi Topic Conference, 2001. IEEE INMIC 2001. Technology for the 21st Century. Proceedings. IEEE International, vol., no., pp.62,68, 2001

[5] X. Hong, K. Xu, M. Gerla, "Scalable routing protocols for mobile ad hoc networks," Network, IEEE, vol.16, no.4, pp.11,21, Jul/Aug 2002

[6] T. Clausen and P. Jacquet, "Optimized Link State Routing Protocol," (3626) , Internet Engineering Task Force , IETF , RFC 3626 (Informational) (2003).

[7] H. Wu, Chunming Qiao, S. De, O. Tonguz, "Integrated cellular and ad hoc relaying systems: iCAR," Selected Areas in Communications, IEEE Journal on , vol.19, no.10, pp.2105,2115, Oct 2001
[8] C. Perkins, E. Belding-Royer, S. Das, "Ad hoc On-Demand Distance Vector (AODV) Routing," (3561) , Internet Engineering Task Force , IETF , RFC 3561 (Informational) (2003).

[9] Z.J. Haas and M.R. Pearlman, "The performance of query control schemes for the zone routing protocol," Networking, IEEE/ACM Transactions on, vol.9, no.4, pp.427,438, Aug 2001

[10] P. Guangyu, M. Geria, Xiaoyan Hong, "LANMAR: landmark routing for large scale wireless ad hoc networks with group mobility," Mobile and Ad Hoc Networking and Computing, 2000. MobiHOC. 2000 First Annual Workshop on , vol., no., pp.11,18, 2000

[11] V. Ramasubramanian, Z.J. Haas, E. Sirer, "SHARP: A hybrid adaptive routing protocol for mobile ad hoc networks," Proceedings of the 4th ACM international symposium on Mobile ad hoc networking \& computing, 2003. vol., no., pp.303,314, 2003

[12] T.A. Ramrekha, G.P. Millar, C. Politis, "A model for designing scalable and efficient adaptive routing approaches in emergency Ad hoc communications," Computers and Communications (ISCC), 2011 IEEE Symposium on, vol., no., pp.916,923, June 28 2011-July 12011

[13] K. Pandey, A. Swaroop, "A Comprehensive Performance Analysis of Proactive, Reactive and Hybrid MANETs Routing Protocols," International Journal of Computer Science Issues (IJCSI), vol.8, no.6, p432, Nov 2011

[14] ns-3. (2012). [online]. Available at: http://www.nsnam.org/.

[15] P.G. Lye, J.C. McEachen, "A Comparison of Optimized Link State Routing with Traditional Routing Protocols in Marine Wireless Ad-hoc and Sensor Networks," System Sciences, 2007. HICSS 2007. 40th Annual Hawaii International Conference on , vol., no., pp.293b,293b, Jan. 2007

[16] Bow-Nan Cheng and S. Moore, "A comparison of MANET routing protocols on airborne tactical networks," MILITARY COMMUNICATIONS CONFERENCE, 2012 - MILCOM 2012 , vol., no., pp.1,6, Oct. 29 2012-Nov. 12012 